\title{
Avaliação de pacientes utilizando equipamento inovador de auxílio à visão subnormal
}

\author{
Evaluation of patients using an innovative low-vision aid
}

\author{
Fernanda Alves da Silva Bonatti ${ }^{1}$ \\ José Américo Bonatti ${ }^{2}$ \\ Marcos Wilson Sampaio ${ }^{3}$ \\ Maria Aparecida Onuki Haddad ${ }^{4}$ \\ Patricia Regina Mucédola de Souza ${ }^{5}$ \\ Newton Kara José ${ }^{6}$
}

\begin{tabular}{|l|}
\hline RESUMO \\
\hline Objetivo: Avaliação preliminar na Clínica Oftalmológica do Hospital das \\
Clínicas da Faculdade de Medicina da Universidade de São Paulo (USP) \\
de equipamento inovador para baixa visão desenvolvido na USP que \\
consiste em prancha de leitura acoplada a lente de aumento que mantém \\
fixos o foco a linha de leitura. Métodos: Foram avaliados 9 pacientes com \\
visão subnormal usando o supracitado equipamento prancha de leitura \\
acoplada a lente para auxílio em visão subnormal desenvolvida na USP em \\
comparação com uso de uma lupa teste manual de dioptria semelhante, \\
considerando-se os seguintes parâmetros de avaliação: causa da doença, \\
acuidade visual corrigida no melhor olho para longe, impressão pessoal do \\
paciente comparando prancha de leitura e lupa teste, impressão dos \\
autores observando o uso dos 2 auxílios acima citados. Resultados: A \\
preferência pelos recursos foi: prancha de leitura acoplada a lupa - 5 \\
pacientes; lupa manual - 2 pacientes; sem preferência por nenhum dos \\
recursos - 1 paciente; inadequados para avaliação dos recursos preferidos \\
- 1 paciente. Conclusão: Neste estudo preliminar, a maioria dos pacientes \\
avaliados preferiu o recurso prancha de leitura acoplada à lupa, o que \\
mostra que este produto inovador facilita a leitura; o médico avaliador \\
interpreta a opinião do paciente como um especialista e contribui para o \\
aprimoramento do produto para que ele possa ser futuramente submetido \\
a novas avaliações.
\end{tabular}

Objetivo: Avaliação preliminar na Clínica Oftalmológica do Hospital das
Clínicas da Faculdade de Medicina da Universidade de São Paulo (USP)
de equipamento inovador para baixa visão desenvolvido na USP que
consiste em prancha de leitura acoplada a lente de aumento que mantém
fixos o foco e linha de leitura. Métodos: Foramavaliados 9 pacientes com
visão subnormal usando o supracitado equipamento prancha de leitura
acoplada a lente para auxílio em visão subnormal desenvolvida na USP em
comparação com uso de uma lupa teste manual de dioptria semelhante,
considerando-se os seguintes parâmetros de avaliação: causa da doença,
acuidade visual corrigida no melhor olho para longe,impressão pessoal do
paciente comparando prancha de leitura e lupa teste, impressão dos
autores observando o uso dos 2 auxílios acima citados. Resultados: A
preferência pelos recursos foi: prancha de leitura acoplada a lupa - 5
pacientes; lupa manual - 2 pacientes; sem preferência por nenhum dos
recursos - 1 paciente; inadequados para avaliação dos recursos preferidos
- 1 paciente. Conclusão: Neste estudo preliminar, a maioria dos pacientes
avaliados preferiu o recurso prancha de leitura acoplada à lupa, o que
mostra que este produto inovador facilita a leitura; o médico avaliador
interpreta a opinião do paciente como um especialista e contribui para o
aprimoramento do produto para que ele possa ser futuramente submetido
a novas avaliações.
Descritores: Baixa visão/reabilitação; Equipamentos e provisões; Desenho de equipamento; Leitura; Auxiliares sensoriais

\section{INTRODUÇÃO}

\footnotetext{
sign e Arquitura) pela Universidade de São t

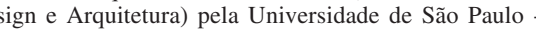
USP - São Paulo (SP) - Brasil.

Doutor em Oftalmologia da Clínica Oftalmológica da Faculdade de Medicina pela USP - São Paulo (SP) Brasil.

Doutor em Oftalmologia da Clínica Oftalmológica da Faculdade de Medicina pela USP - São Paulo (SP) Brasil.

Doutora em Oftalmologia da Clínica Oftalmológica da Faculdade de Medicina pela USP - São Paulo (SP) Brasil.

Ortoptista Doutora em Oftalmologia da Clínica Oftalmológica da Faculdade de Medicina pela USP - São Paulo (SP) - Brasil.

Professor Titular de Oftalmologia da USP - São Paulo (SP) - Brasil e Universidade Estadual de Campinas UNICAMP - Campinas (SP) - Brasil.

Endereço para correspondência: José Américo Bonatti. R. Teodoro Sampaio, 744/105 - São Paulo (SP) CEP 05406-000

E-mail: bonatti@uol.com.br

Recebido para publicação em 12.08.2007

Última versão recebida em 19.11.2007

Aprovação em 11.01.2008 Trabalho realizado no Setor de Visão Sub-Normal da
Clínica Oftalmológica do Hospital das Clínicas da Faculdade de Medicina da Universidade de São Paulo - USP.
} \author{
Leitura; Auxiliares sensoriais
}


população com 65 anos de idade, número que sobe para $20 \%$ da população a partir dos 85 anos (informação verbal) ${ }^{(2)}$.

A Organização Mundial de Saúde (OMS) estimou a existência de 161 milhões de pessoas com deficiências visuais graves no mundo, de acordo com dados baseados na população mundial de 2002; destes, 37 milhões seriam cegos e 124 milhões possuiriam baixa visão ou visão subnormal ${ }^{(3)}$, respectivamente $23 \%$ de cegos e $77 \%$ com baixa visão. De modo geral, para cada pessoa cega há cerca de 3,0 pessoas com baixa visão; existem variações regionais, que se situam entre 2,4 a $5,8^{(4)}$.

O número de cegos foi estimado em $0,4-0,5 \%$ da população, ou seja, de 4 a 5 mil pessoas por milhão de habitantes, porém essa estimativa da prevalência de cegueira no Brasil sofre variações de acordo com os diferentes níveis socioeconômicos do país: $0,25 \%$ em locais semelhantes a países desenvolvidos e $0,75 \%$ em áreas mais pobres economicamente ${ }^{(5)}$. Combinando os dados da OMS com essa estatística brasileira, pode-se inferir que o nosso país, com uma população de 180 milhões, possui 3,6 milhões de pessoas com deficiências visuais graves, dos quais 900 mil são cegos e 2,7 milhões de pessoas apresentam baixa visão.

Existe uma demanda crescente por equipamentos ergonômicos para deficientes visuais, já que eles constituem-se numa população cada vez mais leitora, exigente, ativa e numerosa nos dias atuais, desde os jovens até os idosos. Dentro desta perspectiva, foi desenvolvida uma prancha de leitura acoplada à lupa na Universidade de São Paulo (USP), baseada na inferência das necessidades da população de baixa visão, de modo a permitir que estas pessoas leiam um determinado texto sem perder o foco e a linha de leitura e permitindo que uma das mãos fique livre enquanto a outra empurra a lente para os lados na leitura ${ }^{(6)}$. Este equipamento foi patenteado e premiado como o melhor tema livre no II Congresso Brasileiro de Visão Subnormal, realizado em julho de 2005, e posteriormente teve o relato de sua feitura publicado, demonstrando seu valor intrínseco como produto. Quando se desenvolve qualquer equipamento para uso pessoal deve haver, além da preocupação com sua feitura em si, também a avaliação de sua utilidade e facilidade de uso, ou seja, deve-se testá-lo junto ao seu público alvo, a fim de se estudar a sua real eficácia. Este raciocínio é válido também para equipamento para deficientes. Dentro desta perspectiva este artigo tem como objetivo a avaliação preliminar deste equipamento para a baixa visão desenvolvido na Universidade de São Paulo.

\section{MÉTODOS}

O projeto deste trabalho foi apresentado para a Comissão de Ética em pesquisa do Hospital das Clínicas da FMUSP e aprovado para execução.

Foram avaliados 9 pacientes do serviço de visão Subnormal do Hospital das Clínicas da Faculdade de Medicina da USP com acuidade visual corrigida no melhor olho nas faixas moderada (de 20/80 a 20/160) e grave (de 20/200 a 20/400), em que os auxílios para ampliação da imagem para perto são os mais indicados ${ }^{(7)}$.

Todos os pacientes usaram o equipamento prancha de leitura acoplada à lupa para auxílio em visão subnormal desenvolvida na $\mathrm{USP}^{(6)}$ e uma lupa manual de dioptria semelhante para ler um texto de jornal ou revista e o resultado foi comparado. Foram considerados os seguintes parâmetros de avaliação:

a) causa da doença;

b) acuidade visual corrigida no melhor olho para longe;

c) impressão pessoal do paciente comparando prancha de leitura acoplada à lupa e a lupa manual;

d) impressão dos autores observando o uso pelos pacientes dos dois auxílios acima citados.

\section{RESULTADOS}

Os resultados são relatados a seguir correspondendo aos parâmetros de avaliação propostos em Métodos.

Paciente 1 - 59 anos

a) coriorretinite macular;

b) $20 / 320$;

c) a leitura da tabela com a prancha de leitura acoplada à lupa é mais calma;

d) leu melhor com a prancha de leitura acoplada à lupa, ou seja, com menos dificuldade e com mais tranqüilidade do que com a lupa manual.

Paciente 2 - 83 anos

a) doença macular relacionada à idade (DMRI);

b) $20 / 250$;

c) não viu praticidade na prancha de leitura acoplada à lupa em termos de leitura rápida. Achou lupa manual mais prática. Gostou do posicionamento e da ergonomia, não do tamanho da lente;

d) percebemos que ele se deu bem com o conforto que o equipamento proporciona em termos de inclinação e conforto cervical, mas preferiu a liberdade e rapidez de manuseio da lupa de mão, apesar de haver dificuldade de fixar o foco.

Paciente 3 - 79 anos

a) DMRI;

b) $20 / 200$;

c) leu melhor com a prancha de leitura acoplada à lente de aumento. Viu letras faltando pedacinho. Teve muita dificuldade para achar o foco de leitura com a lupa manual;

d) sentiu maior conforto com a prancha de leitura, pois mantém o foco de leitura fixo. Sentiu dificuldade de manter o foco com a lupa manual.

Paciente 4 - 80 anos

a) DMRI;

b) $20 / 320$;

c) como demora muito para ler, acha mais confortável a lupa presa com o foco fixo da prancha de leitura;

d) prefere a comodidade do foco fixo, que mantém a linha de leitura na prancha acoplada à lupa.

Paciente 5 - 61 anos

a) maculopatia diabética; 
b) não informa visão;

c) não lê de modo fluente;

d) informação não adequada para se considerar a avaliação do equipamento deste trabalho.

Paciente 6 - 13 anos

a) coriorretinite macular;

b) $20 / 160$;

c) achou mais fácil a prancha de leitura acoplada à lupa porque "não precisava se esforçar tanto";

d) leu com menor esforço e por isso de modo mais tranqüilo com a lupa acoplada à prancha de leitura. Sua atenção ficou mais concentrada no texto.

Paciente 7 - 29 anos

a) estrias angióides e degeneração macular bilaterais;

b) $20 / 80$;

c) não viu vantagem com a prancha de leitura acoplada à

lupa. Tanto faz seu uso ou da lupa manual;

d) não é fluente na leitura. Fica procurando a imagem.

Paciente 8 - 56 anos

a) DMRI;

b) $20 / 400$;

c) acha mais fácil ler com a lupa manual;

d) ainda não se habituou ao uso das lupas, vai retornar para treinamento, pois não consegue comparar os dois recursos em termos de facilidade de leitura e conforto. Lê devagar, com dificuldade para entender as palavras.

Paciente 9 - 34 anos

a) coriorretinite macular;

b) $20 / 200$;

c) a prancha de leitura acoplada à lupa é melhor porque com a lupa manual precisa ficar ajustando o foco;

d) leu de maneira mais despreocupada com a prancha de leitura acoplada à lupa.

Resumindo estes resultados, em termos de preferência pelos recursos, temos:

- Preferência pela prancha de leitura acoplada à lupa: 5 pacientes.

- Preferência pela lupa manual: 2 pacientes.

- Indiferença pelos recursos avaliados: 1 paciente.

- Não adequado para avaliação dos recursos preferidos: 1 paciente.

\section{DISCUSSÃO}

Os problemas de visão podem representar prejuízos nas atividades da vida diária de indivíduos com baixa visão, já que cerca de $85 \%$ da interação do homem com o meio se faz pela visão $^{(8)}$. Por isso torna-se essencialmente importante o investimento em equipamentos de auxílio para a baixa visão, pois o uso da correção óptica para erros refracionais poderá não ser suficiente para a melhor resolução visual ${ }^{(9)}$. Em nosso país foram desenvolvidos alguns equipamentos que representaram soluções engenhosas e acessíveis para o paciente com baixa visão, como, por exemplo, os Sistemas Telescópicos de custo reduzido $^{(9-10)}$. A situação econômica de países em desenvolvimento deve ser considerada ao se projetar equipamentos para a sua população. Nessa linha de pensamento foi desenvolvida na USP a prancha de leitura acoplada à lupa ${ }^{(6)}$, que mantém o foco e a linha de leitura fixos, visando o conforto do paciente, objeto da avaliação clínica desse trabalho.

Dentre as experiências brasileiras no desenvolvimento de equipamentos para a área médica podemos citar o pioneirismo do Prof. Dr. Adolfo Leirner na cardiologia, que revelou, em entrevistas concedidas aos autores desse trabalho, no Instituto do Coração (InCor), em São Paulo, nos dias 27 de fevereiro de 2007 e 15 de março de 2007, que compensa, em termos financeiros, a produção nacional em comparação aos similares importados, mais caros. Também merecem ser citadas as experiências da Clínica Oftalmológica da Faculdade de Medicina da USP, onde foram desenvolvidos o crioextrator, o vitreófago, o gancho de íris, o diatermocoagulador bipolar, o oftalmoscópio binocular indireto, o videoceratoscópio e o equipamento de geração e registro de pressão intra-ocular ${ }^{(11-15)}$.

O design de equipamentos médico-hospitalares, para deficientes e para a população dos países em desenvolvimento foi incentivado, a partir da década de 1970, por aqueles que condenavam o design essencialmente consumista ${ }^{(16)}$.

Na década de 1980, quando o designer alemão Gui Bonsiepe veio ao Brasil convidado para implantar, no Conselho Nacional de Desenvolvimento Científico e Tecnológico (CNPq), uma política nacional de inovação, afirmou que o design de produtos para os deficientes físicos não pertencia ao grupo de tecnologia de ponta, não somente no Brasil, mas a nível internacional ${ }^{(17)}$. Uma das causas apontadas para justificar esse fenômeno foi a falta de pressão do mercado, que tende, nos dias atuais, a aumentar, em razão do envelhecimento da população e dos movimentos a favor da inclusão de todos os grupos populacionais ${ }^{(18)}$. A tecnologia brasileira para a fabricação de equipamentos médico-hospitalares ainda está atrasada em relação aos países desenvolvidos. As pesquisas carecem de material humano competente e, principalmente, interessado.

A integração interdisciplinar, condição fundamental para pesquisas nessa área, ainda é ínfima. A “observação participativa”, utilizada na sociologia, é uma metodologia adequada para orientar os profissionais da área da saúde a colherem as informações sobre o uso de equipamentos observando e participando do momento em que os pacientes os estão utilizando ${ }^{(19)}$.

Deve-se ressaltar que avaliação de equipamento para saúde é tema recente e a cada equipamento avaliado acrescentase experiência inestimável neste campo ainda pouco estudado em nosso meio. Insere-se aí, portanto, além da impressão exercida no paciente pelo equipamento, o olhar e opinião do médico sobre o produto avaliado e o que ele observa e interpreta de seu uso pelo paciente a fim de enriquecer esta avaliação, contribuindo para o aprimoramento do instrumental para a saúde em termos de satisfação e melhoria de qualidade de vida do paciente e usuário final, promovendo sua inclusão social de maneira mais eficiente. 
Em termos do número total de sujeitos avaliados, considera-se representativa, para avaliar um produto, uma amostra de indivíduos variando de 5 a $15^{(20)}$, o que ocorreu neste trabalho, em que foram ouvidas nove pessoas. Destas, uma não foi adequada para o estudo. Das oito restantes, cinco apresentaram nítida preferência pelo produto inovador prancha de leitura associada à lupa, duas preferiram a lupa de mão e uma foi indiferente pela preferência de um ou outro recurso de auxílio a visão subnormal. Nota-se, portanto, neste estudo preliminar, que a maioria dos pacientes (5) preferiu o recurso prancha de leitura acoplada à lupa, o que mostra que este produto inovador que fixa a linha de leitura e o foco facilita sobremaneira a leitura para a maioria das pessoas aqui analisadas.

Note-se também, que a opinião do médico avaliador é fundamental para entender e complementar a opinião emitida pelo paciente no sentido de interpretar o que ele disse e acrescentar dados sobre o equipamento avaliado do ponto de vista de alguém especializado naquele produto e mais preparado para traduzir eventuais demandas clínicas que possam aí surgir. É evidente que esta avaliação preliminar pode e deve se estender no futuro, com maior amostragem de indivíduos consultados e mais itens avaliados, até com eventuais aprimoramentos do produto avaliado em função dos dados obtidos neste estudo.

Deve-se destacar, contudo, que como se trata de um equipamento original, ele deveria ser abordado de maneira preliminar para que eventuais questões de praticidade de uso sejam consideradas para que novas avaliações após eventuais modificações possam mais tarde ser feitas.

\section{CONCLUSÃO}

Neste estudo preliminar, a maioria dos pacientes avaliados preferiu o recurso prancha de leitura acoplada à lupa, o que mostra que este produto inovador facilita a leitura. O médico avaliador interpreta a opinião do paciente como um especialista e contribui para o aprimoramento do design do produto para que ele possa ser futuramente submetido a novas avaliações.

\section{ABSTRACT}

Purpose: To perform a preliminary evaluation at the "Clínica Oftalmológica do Hospital das Clínicas da Faculdade de Medicina da Universidade de São Paulo (USP)" of an innovative equipment for low-vision developed at USP containing a reading stand and a magnifier that maintains in a stable position the reading line and focus. Methods: 9 low-vision patients were evaluated using the above mentioned reading stand and a magnifier developed at USP comparing it with a hand magnifier of similar power taking into account the following evaluation parameters: etiology of low-vision, best corrected visual acuity for distance, patient's opinion comparing both low-vision aid resources, authors' opinion observing the patient using both low-vision aids. Results: The numerical preference for the low-vision aids was: 5 patients for the reading stand and a magnifier that maintains at a stable position the reading line and focus; 2 patients for the hand magnifier; 1 patient indifferent regarding any of the resources; 1 patient inadequate for the evaluation of the low-vision aids. Conclusion: This preliminary study shows the preference of the majority of the patients for the reading stand and a magnifier that maintains at a stable position the reading line and focus showing that this innovative product makes reading easier; the evaluator doctor translates the patient's opinion as an expert and contributes to the improvement of the product so that it can be evaluated again in the future.

Keywords: Vision, low/rehabilitation; Equipment and supplies; Equipment design; Reading; Sensory aids

\section{REFERÊNCIAS}

1. CID - Classificação Estatística Internacional de Doenças e Problemas Relacionados à Saúde. 10ª rev. São Paulo: Edusp; 2004. Cap. 7; Doenças do olho e anexos (H00-H59).

2. Visão Subnormal [apostilado]. In: III Curso de Reciclagem em Oftalmologia do Departamento de Oftalmologia do Hospital das Clínicas da Faculdade de Medicina da USP. São Paulo, Maio 8; 2004. São Paulo: Universidade de São Paulo; 2004.

3. World Health Organization (WHO). Fifty-Ninth World Health Assembly. Prevention of avoidable blindness and visual impairment [Internet]. Geneva; WHO; 2006. [cited 2007 Jun 12]. Available from: www.who.int/gb/ebwha/pdf_files/ WHA59/A59_12-en.pdf.

4. Haddad MAO. Habilitação e reabilitação visual de escolares com baixa visão: aspectos médico-sociais [tese]. São Paulo: Faculdade de Medicina, Universidade de São Paulo; 2006.

5. Temporini ER, Kara-José N. A perda da visão: estratégias de prevenção. Arq Bras Oftalmol. 2004;67(4):597-601.

6. Bonatti FA. Desenvolvimento de equipamento de auxílio à visão subnormal Arq Bras Oftalmol. 2006;69(2):221-6.

7. Colenbrander A. Reabilitação de baixa visão. In: Veitzman S. Visão subnormal. São Paulo: Cultura Médica; 2000. p.87-109. [Coleção de Manuais Básicos].

8. Alves MR, Kara-José N. O olho e a visão: o que fazer pela saúde ocular. São Paulo: Vozes, 1996. p.151.

9. Haddad MA, Sampaio MW, Kara-José N. Auxílios para baixa visão. São Paulo: Laramara, 2001.

10. Haddad MAl. Low cost telescopic system: its effectiveness in cases of macular retinochoroiditis due to congenital toxoplasmosis. In: Stuen C, Arditi A, Horowitz A, Lang MA, Rosenthal B, Seidman K, editors. Vision rehabilitation: assessment, intervention, and outcomes. Lisse: Swets \& Zeitlinger; 2000. p.195-9.

11. Suzuki H, Suzuki R, Suzuki CR. Gancho de íris para dilatação mecânica da pupila: modo de construção. Arq Bras Oftalmol. 1995;58(5):367-8.

12. Suzuki H, Suzuki R, Suzuki CR. Diatermo coagulador bipolar coaxial: modo de construção. Arq Bras Oftalmol. 1995;58(3):175-6.

13. Cavalcante I. Ataque à miopia com raio laser. J USP. 1991;5(171):12.

14. Oliveira AC, Yasuoka FM, Tonissi Jr. SA, Cansian AM, Ramos JE, Romão $\mathrm{AC}$, et al. Construção de um vídeokeratoscópio para análise topográfica da córnea [abstract]. In: XV Encontro Nacional de Física da Matéria Condensada, 1992; Caxambu. Programa e Resumos.

15. Bonatti JA, Suzuki H, Kara-José N, Matheus LC. Desenvolvimento de equipamento de geração e registro de pressão intra-ocular suportada por perfuração corneana colada com fibrina. Arq Bras Oftalmol. 1997;60(5):514-5.

16. Papanek V. Diseñar para el mundo real: ecología humana y cambio social. Madrid: Herman Blume; 1977. p.339.

17. Bonsiepe G. Desenho Industrial para pessoas deficientes. Brasília: CNPq, 1982. p.96.

18. Clarkson J, Coleman R, Keates S, Lebbon C, editors. Inclusive design: design for the whole population. London: Springer, 2003. p.608.

19. Margolin V. Um "modelo social" de Design: questões de prática e pesquisa. Design em Foco. 2004;I(1):43-8

20. Keinonen T. Evaluation in Product Development: presenting the draft and prototyping. [place unknown]: 2007. [cited 2007 Mar 3]. Available from: http://www2.uiah.fi/projects/metodi/13c.htm 